\title{
MICROGRID POWER CONVERTER CONTROL WITH SMOOTH TRANSIENT RESPONSE DURING THE CHANGE OF CONNECTION MODE
}

\author{
Gustavo M. S. Azevedo, Marcelo C. Cavalcanti, Francisco A. S. Neves, \\ Leonardo R. Limongi, Fabricio Bradaschia \\ Federal University of Pernambuco (UFPE) \\ Recife - PE, Brazil \\ e-mail: gustavomsa@aim.com, \{marcelo.cavalcanti, fneves\}@ufpe.br
}

\begin{abstract}
Master-slave configuration is a suitable alternative to droop control method to perform the proper power-sharing in microgrids. Microgrids based on master-slave configuration need one master converter (MC) which should be able to work in two operation modes: grid-forming when an outage occurs in the main grid; grid-feeding when the main grid is available. The $\mathrm{MC}$ is responsible to control the microgrid voltage and frequency when it is disconnected from the main grid and it also plays an important role during changes of the microgrid connection mode. This paper presents a control scheme that allows the MC operates in both modes (gridfeeding and grid-forming) and proposes a control system management strategy to manage the MC operation under any microgrid connection mode including the transitions between these modes. As a result, the microgrid transient response is smooth during its change of connection mode. Simulations and experimental results are presented to illustrate the feasibility and performance of the proposed control schemes for the MC.
\end{abstract}

Keywords - Distributed Generation, Grid Synchronization, Microgrids, Parallel Inverters.

\section{INTRODUCTION}

Microgrid has been proposed for effective integration of distributed energy resources (DER), energy storage systems (ESS) and loads to the utility grid, providing high quality and high reliability electric power for the end-users [1], [2]. The microgrid is connected to the main grid at the point of common coupling (PCC), mainly in low-voltage distribution level, but it should also be able to operate without this connection. In such systems, the DER and ESS are connected to the local electric grid through power conditioning units. Thus, inverters or ac-ac converters feed local loads via a common ac bus (the microgrid ac bus).

According to [3], these microgrid converters can be classified into grid-feeding, grid-supporting, and grid-forming power converters depending on their operation mode in a microgrid. The grid-feeding converters are mainly designed to deliver power to an energized grid. They can be represented as an ac current source connected to the grid. The grid-forming converters can be represented as an ac voltage source, setting the voltage amplitude and frequency of the local microgrid by

Manuscript received 03/02/2014; revised 01/06/2014; accepted for publication 19/08/2014, by recommendation of the Special Section Editor Mário L. da S. Martins. using a proper control loop. The grid-supporting converters can be represented either as a current source or as a voltage source, i.e., it is in between a grid-feeding and a grid-forming power converter. Its main objective is to deliver proper values of active and reactive power, contributing to the regulation of the grid frequency and the voltage.

The load power demand of the microgrid must also be shared among the DER and ESS through the proper control of their converters. For this purpose, the droop control method [4]-[6] is typically reported in the literature as a good choice, relying on the fact that it does not require a communication link among the converters [6], [7]. However, this method has several drawbacks, such as [8], [9]: the power sharing is strongly affected by line impedance; there is an inherent tradeoff between the power sharing accuracy and the output voltage regulation. Moreover, it is worth to mention that communication is not necessary for a simple parallel operation of converters (like in distributed uninterruptible power supply systems), but it will be always necessary in microgrids to perform other functionalities apart from power sharing (e.g., changing the reference values of voltage, frequency, active and reactive power) [10], [11]. All converters in a microgrid based on droop control are grid-supporting type and controlled as a voltage source.

On the other hand, the master-slave configuration [12]-[14] is a suitable alternative to droop control method to perform the proper power-sharing in microgrids [8], [15], specially when loads and converters are close to each other, i.e., for short distribution lines [3]. As advantages, it can be mentioned the following: there is no current circulation among converters; the power sharing does not depend on the line impedance; the voltage amplitude and frequency do not exhibit appreciable deviation. This power sharing method needs a low band-width communication link to set the power references for the slave converters. Although the communication link is considered as a drawback [4]-[7], in microgrid applications it is required anyway as mentioned before. A simplified diagram of a microgrid based on the master-slave configuration is shown in Figure 1.

Regarding the master-slave based microgrids, they must operate synchronous with the main grid when in the gridconnected mode. On the other hand, during faults in the main grid, the microgrid disconnects itself and at least one converter must define a reference voltage (both amplitude and frequency) for the whole microgrid. Such converter is also known as the master converter (MC) of the microgrid [12], [13]. The MC must operate as a grid-feeding or gridforming converter depending on the main grid availability. 


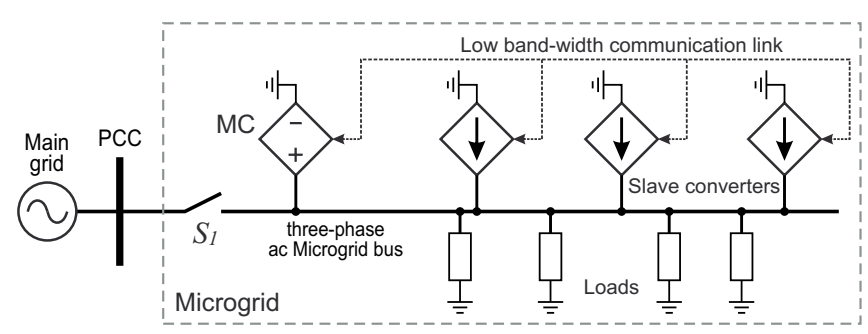

Fig. 1. Simplified diagram of a microgrid based on the master-slave configuration.

Besides, it must have a suitable synchronization system for the grid-connected mode and a sinusoidal oscillator to generate the voltage reference for the islanded autonomous mode. Moreover, the transition between both modes should occur smoothly (without abnormal transients) and as fast as possible, in order to ensure the quality of the electrical energy supplied to the loads connected to the microgrid [16]. During the transition between these two operation modes, the synchronization unit should provide precise synchronization signals that allow the MC to establish a stable voltage in the microgrid. During the reconnection to the main grid, the voltage generated by the MC should be resynchronized with the grid voltage: the synchronization unit varies slowly the phase angle and frequency of the microgrid voltage to resynchronize with the grid voltage. Since all the other converters and equipment connected to the microgrid would be subjected to the reconnection frequency and phase-angle transients, this maneuver must be made in a stable and secure way [3].

A control scheme that allows the MC operates in both modes, grid-feeding and grid-forming, and a management strategy for this control are proposed in this paper. Such scheme comprises of the current and voltage control loops, a single oscillator/synchronization system unit and a control management unit. The aim is comply with the MC requirements that are mentioned above. This work focus mainly on these MC control units and it does not dedicate much attention to the microgrid overall control as well as the converters primary sources (connected to the converters' dc link). This paper is an extended version of [15], with an improved description of the MC operation management and additional analyses. This paper is organized as follows: Section II presents a short description of the converter current and voltage controllers in stationary reference frame. It is pointed out in which condition these controllers must be used in accordance with the main grid state. In Section III it is presented a way to obtain both voltage and current references for each converter operation mode (grid-feeding or grid-forming). The transition between both operation modes is also described. Finally, the performance of the proposed control scheme and its simulation and experimental evaluation are discussed and reported.

\section{INVERTER CONTROL STRATEGIES}

The control of the MC depends on the connection of the microgrid to the main grid (grid-connected operation or island mode). When the main grid is available, the microgrid voltage is defined by the main grid and the MC is controlled in such a way to inject or absorb power (in according to the primary source needs). Therefore, it is more convenient that the $\mathrm{MC}$ operates as a controlled current source, i.e., as a gridfeeding power converter. However, when the main grid is not available, the MC is responsible to impose the microgrid voltage and it has to be controlled as a voltage source, acting as a grid-forming converter. Both operation modes are achieved by the same voltage-source inverter (VSI) controlled as a voltage or as a current source.

The electrical scheme of a VSI and its output LC filter are shown in Figure 2. $R_{1}$ represents the equivalent series resistance (ESR) of $L_{1}$. The ESR of $C_{f}$ is neglected. It is supposed that there is a primary source of power connected to the dc side of the VSI (not shown in the figure) and that $v_{d c}$ is constant. The switches are controlled by a pulse width modulation (PWM) technique which results in the VSI output voltage vector $\vec{v}_{i}=\left[\begin{array}{lll}v_{i a} & v_{i b} & v_{i c}\end{array}\right]^{T}$ and output current vector $\vec{i}_{i}=\left[\begin{array}{lll}i_{i a} & i_{i b} & i_{i c}\end{array}\right]^{T}$. The LC filter output voltage is $\vec{v}_{o}=$ $\left[\begin{array}{lll}v_{o a} & v_{o b} & v_{o c}\end{array}\right]^{T}$, which corresponds to the microgrid ac bus voltage, and the current injected in the microgrid is $\vec{i}_{o}=$ $\left[\begin{array}{lll}i_{o_{a}} & i_{o_{b}} & i_{o_{c}}\end{array}\right]^{T}$. All these variables can be represented in the stationary reference frame through the Clarke transformation and they will be denoted by the subscript $\alpha \beta$.

The converter output voltage (voltage across the capacitors $C_{f}$ ) can be controlled through the current $\vec{i}_{i_{\alpha \beta}}$, being $\vec{i}_{o_{\alpha \beta}}$ considered as a perturbation. The current $\vec{i}_{i_{\alpha \beta}}$ can be imposed by $\vec{v}_{i_{\alpha \beta}}$ through the inverter switches control. Therefore, the output voltage control can be obtained by two control loops: a current inner loop and a voltage outer loop. Note that the load current is a disturbance of the voltage loop, as well the output voltage is a disturbance of the current loop. However, they can be properly compensated since these quantities are measured.

It is also possible to control the output current, $\vec{i}_{o_{\alpha \beta}}$, through the control of $\vec{i}_{i_{\alpha \beta}}$, since the capacitive current (through $C_{f}$ ) is known. The current $\vec{i}_{i_{\alpha \beta}}$ can be imposed by $\vec{v}_{i_{\alpha \beta}}$ through the inverter switches control, in a similar way to the first case.

\section{A. Current Controller}

The current control loop diagram and the model of the system to be controlled are shown in Figure 3 [15]. It is important to note that the $\alpha$ and $\beta$ components are decoupled and the structure shown in Figure 3 is the same for each component [3]. The PWM gain is considered unitary. The voltage $\vec{v}_{o_{\alpha \beta}}$ added to the controller output is a feedforward

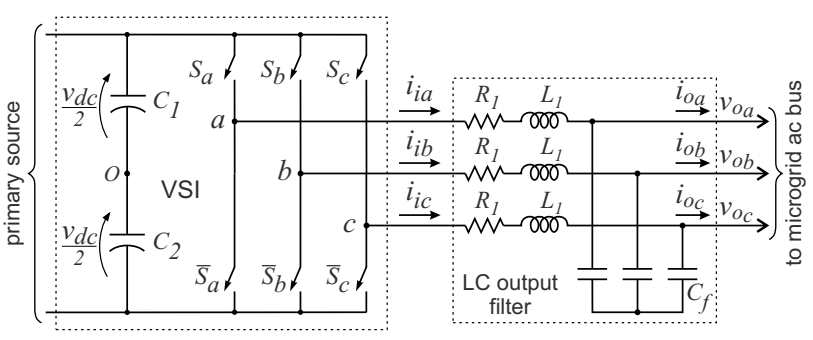

Fig. 2. Electrical scheme of the VSI with the output filter. 


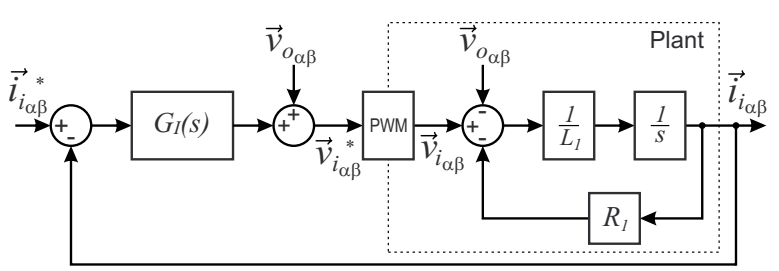

Fig. 3. Current control loop of the VSI.

to improve the system transient response [17]. This voltage is obtained directly from the voltage measurement of the capacitors $C_{f}$. As the currents to be controlled in $\alpha \beta$ are sinusoidal (grid frequency), the proportional-resonant (PR) controller is a good choice [18]. Its transfer function is given by

$$
G_{I}(s)=k_{p_{I}}+k_{r_{I}} \frac{2 s}{s^{2}+\omega^{2}}
$$

where $k_{p_{I}}$ and $k_{r_{I}}$ are the proportional and resonant gains, respectively, and $\omega$ is the controller resonant frequency that must coincide with the fundamental frequency of the signal to be controlled [18], [19].

\section{B. Voltage Controller}

When the converter in Figure 2 is operating in island mode (without the main grid), the voltages across the capacitors $C_{f}$ are undefined and the inverter must be controlled to impose these voltages, which will correspond to the microgrid voltages. This control is made by a voltage outer loop that actuates on the current $\vec{i}_{i_{\alpha \beta} \beta}$. The voltage control loop diagram and the model of the system to be controlled are shown in Figure 4. The feedforward voltage of the current loop should be changed to the reference voltages when the inverter is operating with the voltage control. Thus, the voltage reference is directly imposed to the PWM and the control loops are responsible only to compensate possible deviations [15].

The voltage loop also uses a PR controller with transfer function $G_{V}(s)$ similar to (1), however with different gains.

\section{MASTER CONVERTER OPERATION MANAGEMENT}

When the main grid is available and the voltage and frequency are within the established power quality limits, the microgrid stays connected to the grid through the PCC. In this operation mode, the DER and ESS supply the loads and the power deficit is supplied from the grid. In case of the generated power be higher than the load demand, the

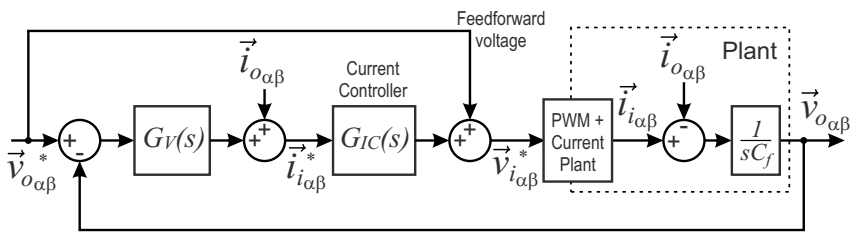

Fig. 4. Voltage control loop of the VSI. microgrid supplies energy to the main grid. Independently of the power flux, the main grid defines the voltage and frequency of the microgrid. Therefore, the MC must follow the voltage and frequency and inject or absorb power in according to the optimal load dispatch. To attend this objective, the MC operates as a grid-feeding power converter. The active and reactive power references, $P^{*}$ and $Q^{*}$, are defined by the microgrid hierarchical controller and passed to the MC through a communication link. The MC current references are calculated based on these power references. A synchronization system is necessary to deliver the power accurately.

When the main grid is not available, due to a fault or a noncompliant power quality requirement, the microgrid is disconnected from the main grid and it starts to operate in an autonomous way (island mode). In this operation mode, all the loads in the microgrid are supplied by the DER and ESS. If the microgrid available power is not enough to supply the load demanded, the non-priority loads must be disconnected, otherwise the overall microgrid will shut down. These sources are current controlled and they need a voltage source to operate. Since the main grid is not available, the $\mathrm{MC}$ is responsible for assuming the voltage and frequency of the microgrid and it operates as a grid-forming converter. Therefore, the MC must be voltage controlled when the microgrid operates in island mode. The voltages references with the desired amplitude and frequency can be obtained by an oscillator. However, this oscillator must be able to resynchronize with the grid voltage during the reconnection process.

The algorithm proposed in [20], [21] can be used to perform the fast detection of the main grid fault condition. The output of this algorithm is used to trigger the disconnection or reconnection procedures, which are presented in the following. The computation of the reference signals for each microgrid operation mode is also discussed.

\section{A. Grid-Connected Operation Mode}

The current controller shown in Section II.A is used in this mode of operation. A strategy that results in sinusoidal and balanced currents is called balanced positive-sequence control (BPSC) [22]. For this strategy, the currents are calculated by

$$
\vec{i}_{i_{\alpha \beta}}^{*}=\underbrace{\frac{2}{3} \frac{P^{*}}{\left|\vec{v}_{\alpha \beta}^{+}\right|^{2}} \vec{v}_{\alpha \beta}^{+}}_{\vec{i}_{P_{\alpha \beta}}^{*}}+\underbrace{\frac{2}{3} \frac{Q^{*}}{\left|\vec{v}_{\alpha \beta_{\perp}}^{+}\right|^{2}} \vec{v}_{\alpha \beta_{\perp}}^{+}}_{\vec{i}_{Q_{\alpha \beta}^{*}}}
$$

where $\vec{i}_{P_{\alpha \beta}}^{*}$ and $\vec{i}_{Q_{\alpha \beta}}^{*}$ represent the current components responsible to produce the active and reactive power, respectively.

In this case, only the positive-sequence component of the voltage at the PCC $\left(\vec{v}_{\alpha \beta}^{+}\right)$is used to calculate the currents. Note that $\vec{v}_{\alpha \beta_{\perp}}^{+}$is only a $90^{\circ}$ phase-shift version of $\vec{v}_{\alpha \beta}^{+}$. Moreover, the terms $\left|\vec{v}_{\alpha \beta}^{+}\right|^{2}$ and $\left|\vec{v}_{\alpha \beta_{\perp}}^{+}\right|^{2}$ in (2) and the amplitude of the vectors $\vec{v}_{\alpha \beta}^{+}$and $\vec{v}_{\alpha \beta_{\perp}}^{+}$are constant, resulting in a sinusoidal current reference of positive-sequence as well.

The reference current calculation through the BPSC strategy requires the estimation of $\vec{v}_{\alpha \beta}^{+}$. The detection 
algorithm of the positive-sequence component presented in [23], called dual second-order generalized integrator - frequency-locked loop (DSOGI-FLL), has a good performance and some characteristics that are very suitable for the control of the MC, as described in [15]. The overall block diagram of the DSOGI-FLL is shown in Figure 5.a. Two SOGI for quadrature signal generation (SOGI-QSG) are used for each voltage component and their output signals are used by the positive-sequence calculator (PSC) to estimate $\vec{v}_{\alpha \beta}^{+}$. The internal block diagram of the DGOGI is shown in Figure 5.b where the gain $k_{s}$ can be seen. This parameter defines the DSOGI dynamics. Similarly, the FLL also has a gain which defines its dynamic behavior. Reference [24] presents a method to tune these parameters.

\section{B. Island Operation Mode}

The voltage controller shown in Section II.B is used in this mode of operation. The point is how to obtain the three-phase voltage references (in $\alpha \beta$ frame) with nominal amplitude and frequency, apart from allowing an easy resynchronization when the grid is restored. The DSOGI-FLL can be used for this purpose if some modifications are made. The shaded box in Figure 5.b highlights the modification. The FLL must be also modified such as presented in [15].

The DSOGI-FLL acts as a synchronization system (its normal function) if $S_{S}$ is in position 0 , otherwise it acts as an oscillator. The amplitude of the generated signal, in the oscillator mode, can be adjusted through the input $c$ given by

$$
c=k_{p}\left(\hat{V}^{*}-\hat{V}^{+}\right)=k_{p}\left(\hat{V}^{*}-\sqrt{v_{\alpha}^{+^{2}}+v_{\beta}^{+^{2}}}\right)
$$

where $k_{p}$ is the proportional gain, $\hat{V}^{*}$ is the reference amplitude and $\hat{V}^{+}$is the amplitude of the DSOGI-FLL output [8].

Assuming that $S_{S}$ changes from 0 to 1 in $t=t_{0}$ and $c$ is kept in 0 , the output $v^{\prime}$ will be described by the $2^{n d}$-order
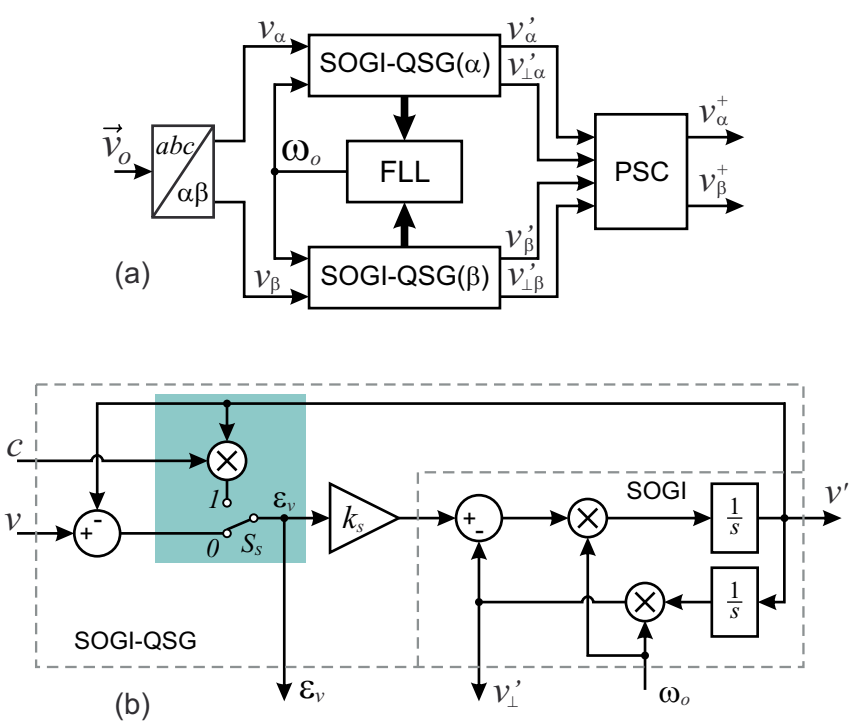

Fig. 5. Simplified block diagram of the DSOGI-FLL with oscillator feature. (a) overall structure, (b) detail of the SOGI-QSG block. differential equation,

$$
\frac{d^{2} v^{\prime}(t)}{d t^{2}}+\omega_{o}^{2} v^{\prime}(t)=0, \quad \text { for } \quad t \geq t_{0}
$$

which the solution is of the type

$$
v^{\prime}(t)=A_{1} \cos \left(\omega_{o} t\right)+A_{2} \sin \left(\omega_{o} t\right)
$$

where $A_{1}$ and $A_{2}$ are complex or real constants. For the other output,

$$
\begin{aligned}
v_{\perp}^{\prime}(t) & =-\frac{1}{\omega_{o}} \frac{d v^{\prime}(t)}{d t} \\
& =A_{1} \sin \left(\omega_{o} t\right)-A_{2} \cos \left(\omega_{o} t\right) .
\end{aligned}
$$

Assuming that the SOGI was operating as synchronization system prior to the $S_{S}$ switching, their outputs are given by

$$
\begin{aligned}
v^{\prime}(t) & =A \cos (\omega t) \\
v_{\perp}^{\prime}(t) & =A \sin (\omega t), \quad \text { for } \quad t \leq t_{0}
\end{aligned}
$$

where $A$ is the voltage amplitude of the DSOGI-FLL output.

At $t=t_{0},(5)$ and (7), as well as (6) and (8), must be equal. Thus, it results

$$
\begin{aligned}
& A_{1}=A \cos \psi \\
& A_{2}=-A \sin \psi
\end{aligned}
$$

where $\psi=\left(\omega-\omega_{0}\right) t_{0}$. Substituting these constants in (5) and (6) results

$$
\begin{aligned}
v^{\prime}(t) & =A \cos \left(\omega_{o} t+\psi\right) \\
v_{\perp}^{\prime}(t) & =A \sin \left(\omega_{o} t+\psi\right), \quad \text { for } \quad t \geq t_{0} .
\end{aligned}
$$

Therefore, if $S_{S}$ changes to 1 , this system will oscillate indefinitely regardless of its input. The amplitude and frequency of the DSOGI output signal will be equal to the values immediately before that change (the same is valid for the PSC output). For this reason, $c$ must be used to restore the amplitude to the rated value. The oscillator frequency can be adjusted any time through $\omega_{o}$.

\section{Transition from Grid-Connected to Island Mode}

The microgrid disconnection procedure is summarized in the flowchart shown in Figure 6.a. When the disconnection is started (e.g., due to a fault), the first action is send a signal to open the microgrid switch $\left(S_{1}\right.$ in Figure 7$)$. After a while, the converter control changes from grid-feeding to grid-forming. It is waited for a while again and then the DSOGI-FLL is changed to oscillator mode. The modified DSOGI-FLL structure (Figure 5) must be used to ensure that the voltage amplitude and frequency given by this oscillator can be restored to their rated values. This structure presents a seamless transition throughout the change from synchronization mode to oscillator mode and vice-versa.

When the procedure is finished, the converter is operating in grid-forming mode following the voltage reference given by the DSOGI-FLL which is operating as a three-phase oscillator. Note that the microgrid voltages are different from the grid voltages, thus it is necessary to keep monitoring the grid to identify when it will be available again and start the reconnection procedure. 


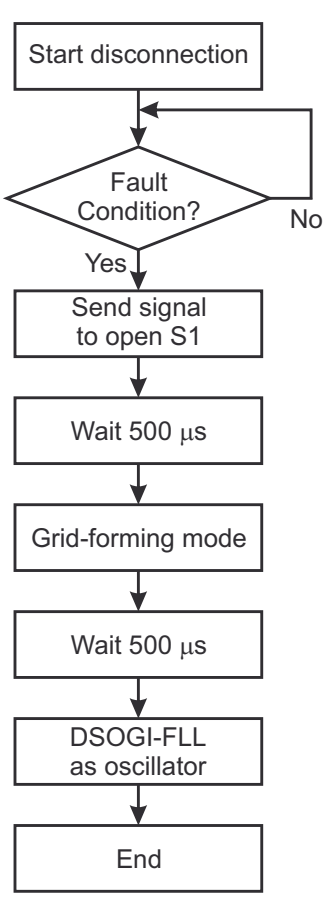

(a)

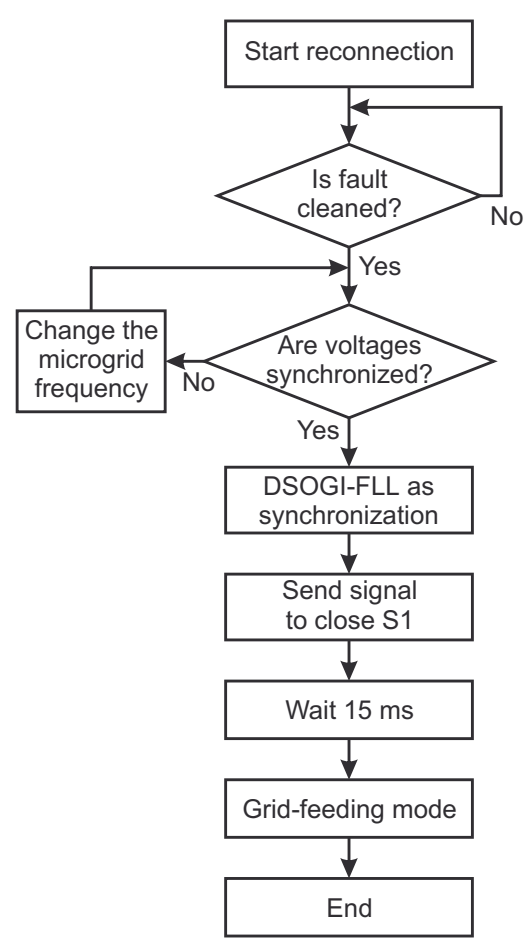

(b)
Fig. 6. Flowchart of the procedures for (a) disconnection and (b) reconnection to the main grid.

\section{Transition from Island to Grid-Connected Mode}

The transition from the island mode to the grid-connected mode occurs, for example, after a fault clearance. It is necessary to resynchronize the microgrid voltages with the main grid before reconnect them. When the grid voltage is restored, the DSOGI-FLL could be enabled again to operate as a synchronization system (it is made by restoring the position of the DSOGI switch, $S_{S}$, shown in Figure 5). However, it can result in an abrupt frequency deviation of the microgrid (including their loads) if the phase shift between both voltages are high. Such frequency deviation can destabilize the overall microgrid. Thus, to overcome this drawback, the DSOGIFLL is kept operating as an oscillator and its frequency is slowly increased (or decreased) to accelerate (or decelerate) the microgrid voltage until it get synchronized with the grid. When the voltages are almost synchronized (both in phase and amplitude), the DSOGI-FLL can be enabled to operate as a synchronization system. Then, the signal to close the microgrid switch is sent. After waiting enough time to ensure that the switch was closed, the converter control changes from grid-forming to grid-feeding. This procedure is summarized in the flowchart shown in Figure 6.b.

\section{SIMULATION RESULTS}

The diagram of the system used for simulation is shown in Figure 7. The system parameters are shown in Table I. The electrical system of medium voltage is modeled by a Thevenin equivalent circuit with rated voltage of $13.8 \mathrm{kV}$ and short circuit power of $100 M V A$. The microgrid is connected to the medium voltage grid through a $50 M V A D y 11$ transformer. In this transformer is also connected another load apart from the microgrid. For simplification purpose, the microgrid is composed only by the MC, a grid-feeding power converter and a three-phase $R L$ load that demands $S=12 k W+j 3 k V A R$ in $380 \mathrm{~V}$. The grid-feeding converter is current controlled and it uses the same control scheme described in Section III.A. The power delivered by this converter is kept constant in $P_{D G}=6 \mathrm{~kW}$ and $Q_{D G}=1.5 \mathrm{kVAR}$.

Initially, the system operates in normal conditions with the MC operating as a grid-feeding converter and supplying $4 k W$ of active power $(1 / 3$ of the rated load) and $1.5 k V A R$ of reactive power. Then, the grid supplies the remaining $2 k W$ with unitary power factor. A phase-to-phase shortcircuit between the phases $b$ and $c$ (through a $5 \Omega$ resistance) in the medium voltage side occurs in $t=50 \mathrm{~ms}$. After $11.6 \mathrm{~ms}$, the fault detection system senses the disturbance and it starts the disconnection procedure according to described in Section III.C. The first action of this procedure is to open $S_{1}$ (Figure 7). It was considered that the switch opens $1 \mathrm{~ms}$ after the signal has been sent. When the disconnection procedure finishes, the MC operates with the voltage control to impose the microgrid voltage and share the load only with the gridfeeding converter. The power delivered by the MC will depend on the load demand. In this study case, the the power are $P_{M C}=P_{D G}=6 \mathrm{~kW}$ and $Q_{M C}=Q_{D G}=1.5 \mathrm{kVAR}$.

The pre-fault and post-fault voltages in the PCC are shown in the first graphic of Figure 8. It is also indicated the moments when the fault occurs and when the $S_{1}$ is opened. The microgrid voltages and its positive-sequence voltage module $\left(\left|\vec{v}_{O_{\alpha \beta}}^{+}\right|\right)$are presented in the second graphic of this figure, where it is possible to see that after the microgrid disconnection, the voltage is reestablished to the rated value by the MC actuation. The microgrid voltage reaches the steadystate in approximately three cycles as indicated in Figure 8. The grid side and MC output currents are also shown in this figure. Note that during the beginning of the fault until $S_{1}$ opens, the microgrid is connected to the grid and, therefore, it is submitted to the grid disturbances.

When the fault is cleared, the fault detection algorithm takes a few milliseconds to detect it and starts the reconnection procedure according to described in Section III.D. The grid and MC voltages as well as their currents, throughout the reconnection steps, are shown in Figure 9. At the end of this

TABLE I

System parameter used in simulation

\begin{tabular}{lc}
\hline Parameter & Value \\
\hline Filter inductance, $L_{1}$ & $2 \mathrm{mH}$ \\
\hline Equivalent resistance of the inductor, $R_{1}$ & $0.1 \Omega$ \\
\hline Filter capacitance, $C_{f}$ & $15 \mu \mathrm{F}$ \\
\hline Microgrid nominal voltage, $V_{n}$ & $380 \mathrm{~V}$ \\
\hline Dc-link voltage, $v_{d c}$ & $650 \mathrm{~V}$ \\
\hline Switching and sampling frequency, $f_{s}$ & $20 \mathrm{kHz}$ \\
\hline Proportional gain of current controller, $k_{p_{I}}$ & 25 \\
\hline Resonant gain of current controller, $k_{r_{I}}$ & 1000 \\
\hline Proportional gain of voltage controller, $k_{p_{V}}$ & 0.02 \\
\hline Resonant gain of voltage controller, $k_{r_{V}}$ & 5 \\
\hline SOGI-QSG gain, $k_{s}$ & $\sqrt{2}$ \\
\hline SOGI-QSG voltage restorer gain, $k_{p}$ & 0.005 \\
\hline
\end{tabular}




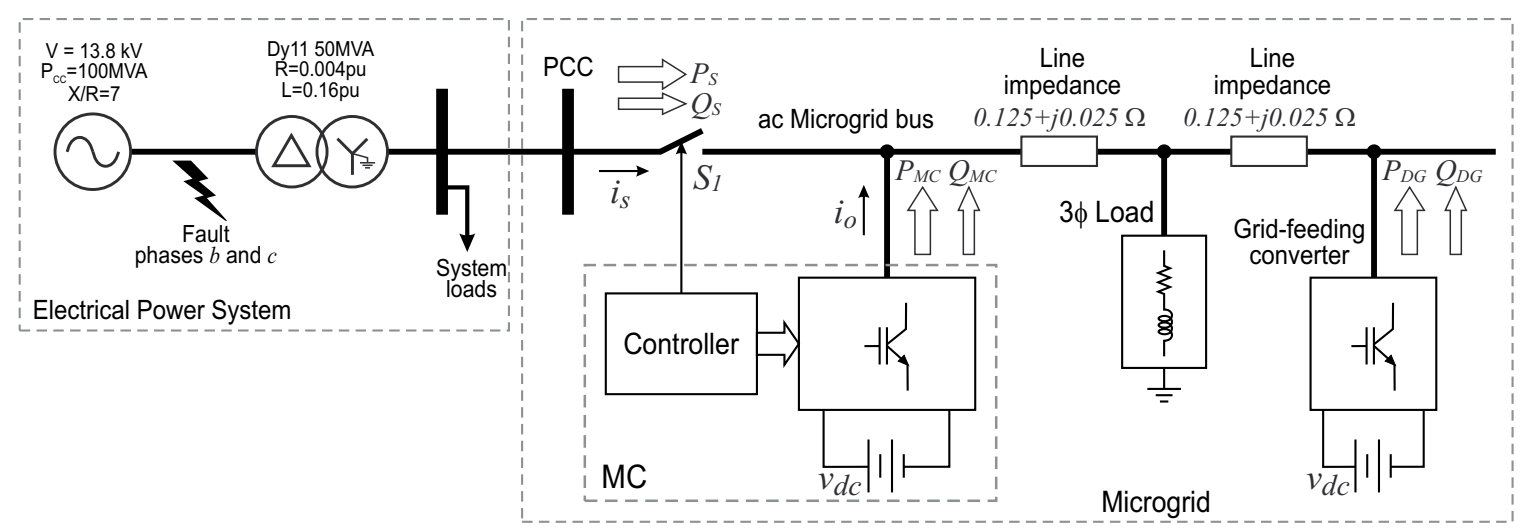

Fig. 7. System scheme used in simulation for evaluation of the MC behavior during microgrid disconnection from and reconnection to the main grid due to an unbalanced fault in the grid.

procedure, the power delivered by the MC is restored to the pre-fault value.

Figure 10 shows a detailed view of the phase $a$ voltages of the grid $\left(v_{a}\right)$ and the microgrid (actually, the MC output voltage $v_{o a}$ ) throughout the synchronization process. The beginning of the synchronization occurs at the same time that the normal condition is detected. In such example, the phase shift between these voltages is small and, therefore, the synchronization is fast. The worst case occurs when the voltages are completely out of phase, which will require more time.
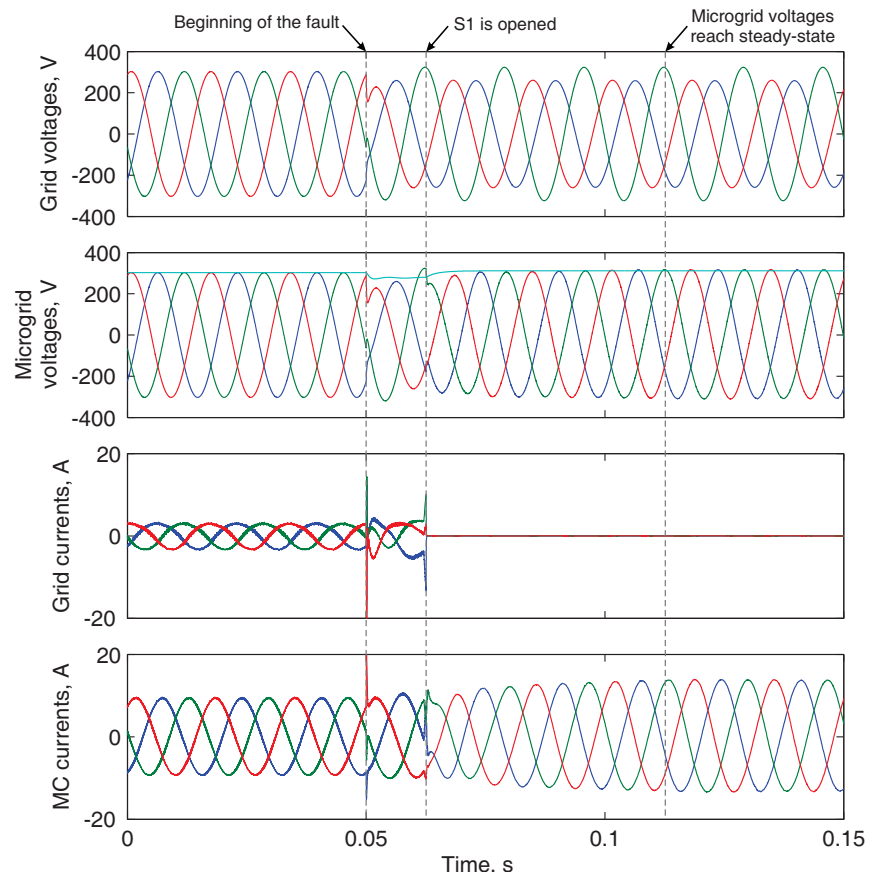

Fig. 8. Behavior of voltages and currents in the electrical system and microgrid during a phase-to-phase fault in the main grid with the disconnection of the microgrid.
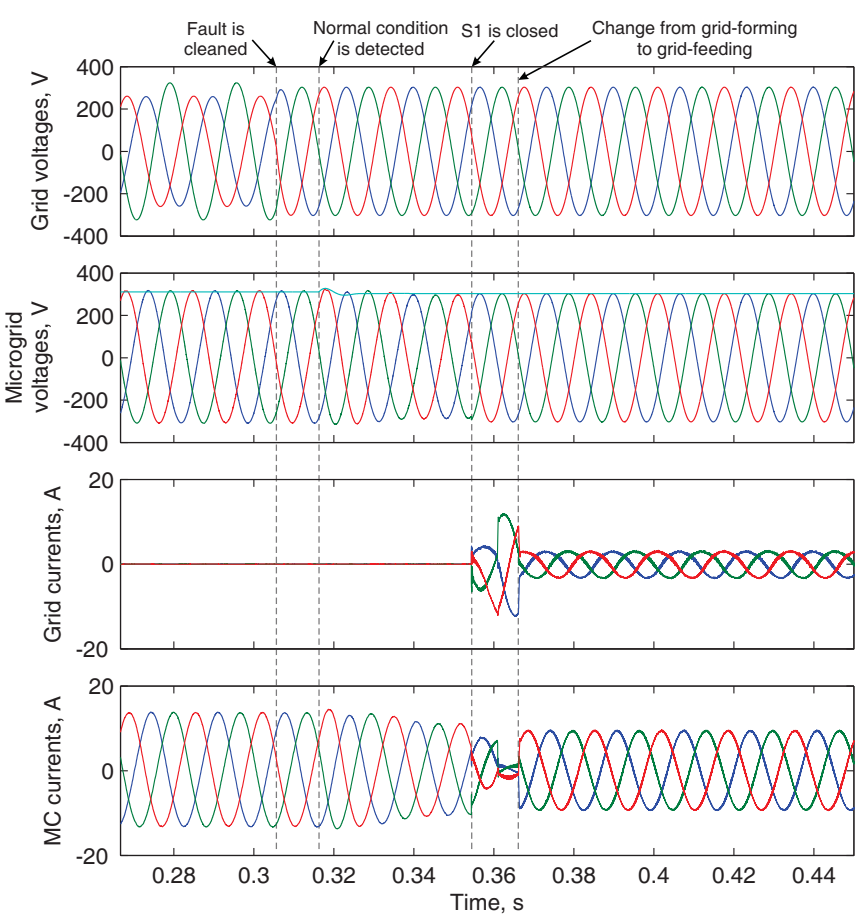

Fig. 9. Behavior of voltages and currents in the electrical system and microgrid during the reconnection to the main grid.

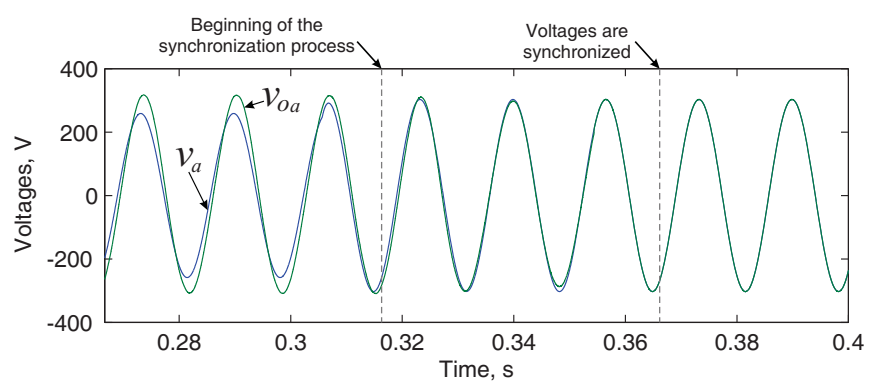

Fig. 10. Phase $a$ voltages of the grid and microgrid during the synchronization process. 


\section{EXPERIMENTAL RESULTS}

A prototype, with a structure similar to that one shown in Figure 7, was developed. The grid-feeding power converter was not yet performed in this prototype and the load is purely resistive. The fault effect is emulated by means of a transformer with taps connected to a second $D y$ transformer. Thus, a single-phase voltage sag in the first transformer will result in a two phase disturbance in the microgrid with voltages similar to that one of the simulation.

In the first experiment, it is evaluated the behavior of the MC during the microgrid disconnection due to a disturbance on the main grid. The disconnection process of the microgrid is started when any abnormality in the PCC voltages is detected. This possible abnormality is determined by the fault detector algorithm, that will begin the disconnection procedure. In the results presented in Figure 11, the event that leads to disconnection is a phase-to-ground fault in the delta $(\Delta)$ side of the transformer (Figure 7), which results in a two phase disturbance in the microgrid PCC.

The three-phase voltages in the PCC and in the microgrid (MC output voltages) are shown in Figure 11. In the first $100 \mathrm{~ms}$, the microgrid is connected to the main grid and their voltages are the same. When the fault occurs, the voltages become unbalanced. Observe that in this moment the system has not yet detected the fault and the microgrid is under the condition of unbalanced voltages. However, the fault detector actuates rapidly, beginning the disconnection procedure. The tripping signal sent to the $S_{1}$ coincides with the instant of fault detection as shown in Figure 11. The complete disconnection process takes around one cycle. When the disconnection is completed, the microgrid voltages become balanced and their amplitudes are recovered to the rated value.

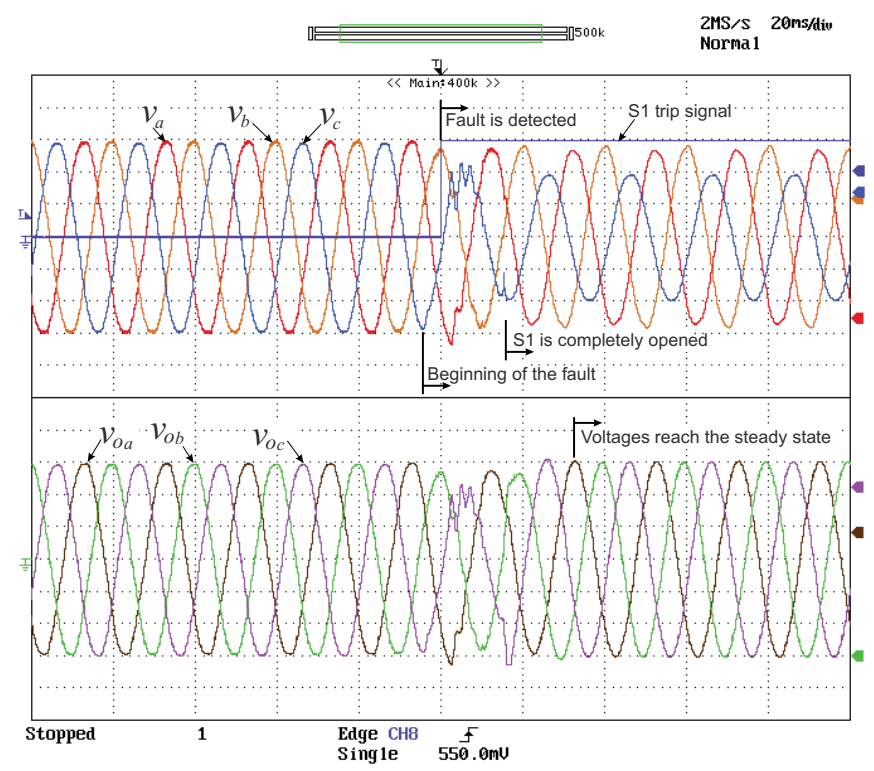

Fig. 11. Transient voltages in the PCC and in the microgrid during a disconnection of the microgrid due to a fault. Scale: $100 \mathrm{~V} / \mathrm{div}$.
The behavior of the MC current, $i_{o_{a}}$, and load current, $i_{L_{a}}$, during the disconnection are depicted in Figure 12. This load current is measured directly on the load, since there is only one load and there are no slave converters. Previous to the fault, the current injected by the converter is higher than the load current, then the surplus power is injected on the grid. The current $i_{o_{a}}$ presents some amount of distortion when the microgrid is connected to the main grid due to the harmonics present in the grid voltages of the lab, which interacts with the LC filter capacitor. The fault leads to a small transient in the MC current which is followed by a second transient when $S_{1}$ is opened. The microgrid voltage and the load current also present a small transient due to the grid voltage disturbances caused by the fault. The MC current becomes equal to the load current when the disconnection is completed.

The next result, shown in Figure 13, presents the synchronization process that takes place after the fault detector notice that the fault was cleared. In this experiment, the grid voltages and microgrid voltages are completely out of phase when the grid is restored. For this reason, the synchronization leads more time to be completed. In this particular case, the synchronization process has led almost $780 \mathrm{~ms}$. When the voltages are synchronized both in phase and amplitude, $S_{1}$ is closed and the MC controller changes to the grid-feeding converter mode.

The currents behavior during the reconnection is shown in Figure 14. The MC current spikes that occurred before $S_{1}$ gets completely closed, is due to the fact that it was used an electromechanical switch (an ac contactor) instead of a static switch as done in simulation. After $S_{1}$ is closed, the MC current has a transient and tend to zero. It occurs because $P^{*}$ and $Q^{*}$ are reset to zero previous to the change to gridfeeding mode in order to reduce the transient current. After

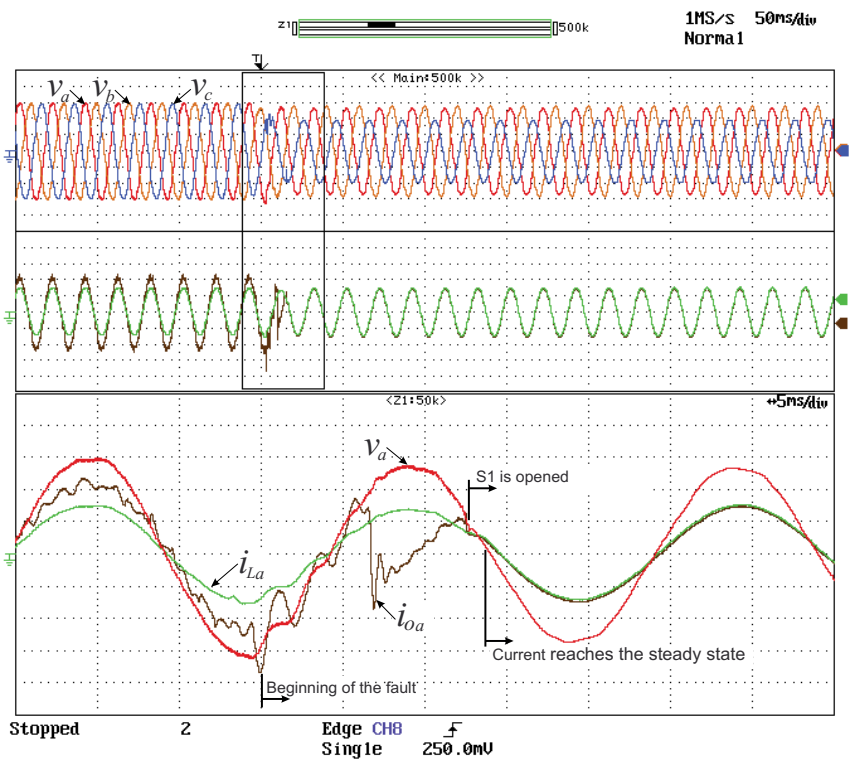

Fig. 12. Transient of the currents during the disconnection of the microgrid. Voltage scale: $100 \mathrm{~V} /$ div, current scale: $1 A / d i v$. 


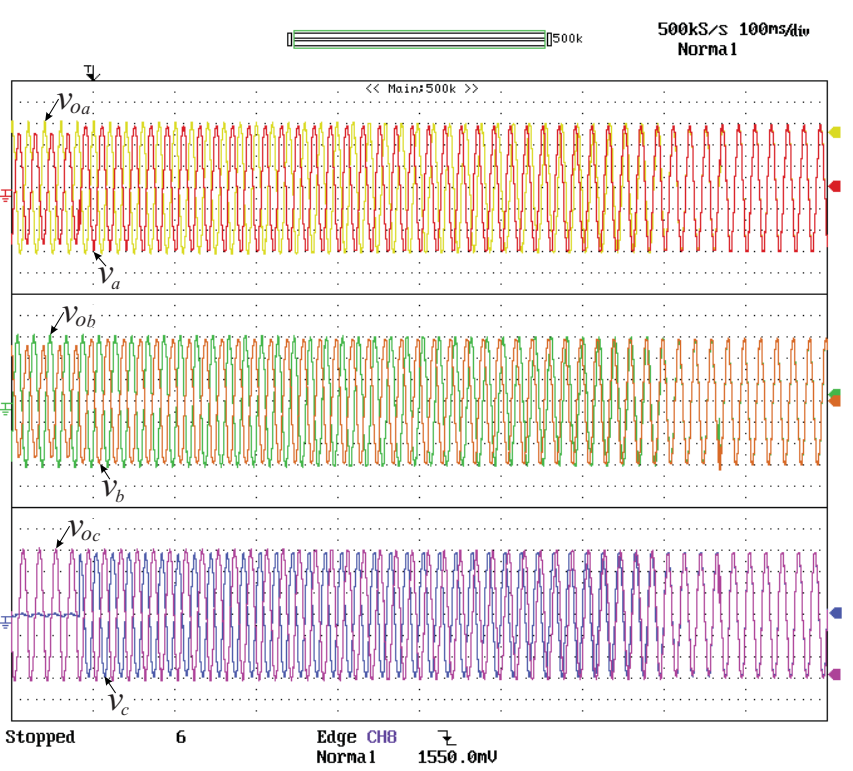

Fig. 13. Voltages in the PCC and in the microgrid during the synchronization procedure. Scale: $100 \mathrm{~V} /$ div.

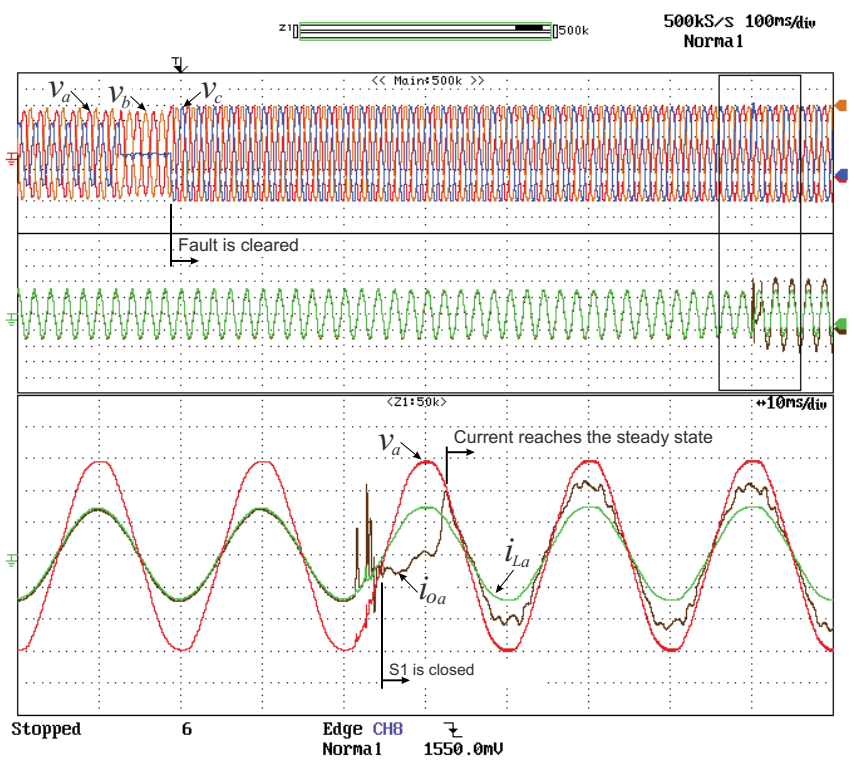

Fig. 14. Transient of the currents during the reconnection. Voltage scale: $100 \mathrm{~V} /$ div, current scale: $1 \mathrm{~A} / \mathrm{div}$.

a while, those power references are restored to their previous values before the microgrid disconnection. Note that in both disconnection and reconnection procedures, the microgrid voltages and the MC output currents change smoothly proving the effectiveness of the proposed control system management strategy for the MC.

\section{CONCLUSION}

This paper presents the current and voltage controllers for a three-phase converter, as well as, a control structure that permits this converter acts as a grid-feeding and a grid-forming power converter for microgrids. Also, it is presented how to obtain the reference voltages or currents depending on the microgrid operation mode. The adaptation of the DSOGIFLL structure permits its operation as a grid synchronization system and as an oscillator. The latter feature is very suitable to generate the output reference voltages of the master converter when the microgrid is operating in island mode. The system can change from synchronization mode to oscillator mode and vice-versa, achieving seamless transient operation between the microgrid modes with fast dynamic responses. The proposed system avoids the well-know high frequency deviation of the microgrid during the reconnection procedure. The simulation and experimental results prove the effectiveness of the proposed control system, guaranteeing the safe operation of the microgrid.

\section{ACKNOWLEDGEMENT}

This work was supported by the "Coordenação de Aperfeiçoamento de Pessoal de Nível Superior" (CAPES) and by the "Conselho Nacional de Desenvolvimento Científico e Tecnológico" (CNPq), Brazil. The authors also would like to thanks the Renewable Electrical Energy Systems (REES) research group at the Technical University of Catalonia (UPC), Spain, for their support in the experiments.

\section{REFERENCES}

[1] R. H. Lasseter, "Microgrids", in 2002 IEEE Power Engineering Society Winter Meeting, pp. 305-308, 2002.

[2] P. T. Cheng, T. L. Lee, C. T. Lee, "An autonomous harmonic filtering strategy for distributed energy resources converters in microgrid", Eletronica de Potencia SOBRAEP, vol. 14, no. 4, pp. 233-240, Dec/2009.

[3] J. Rocabert, A. Luna, F. Blaabjerg, P. Rodríguez, "Control of power converters in ac microgrids", IEEE Transactions on Power Electronincs, vol. 27, no. 11, pp. 4734-4749, Nov/2012.

[4] A. Tuladhar, H. Jin, T. Unger, K. Mauch, "Control of parallel inverters in distributed ac power systems with consideration of line impedance effect", IEEE Transactions on Industry Applications, vol. 36, no. 1, pp. 131-138, Jan/Feb 2000.

[5] J. M. Guerrero, L. G. de Vicuna, J. Matas, M. Castilla, J. Miret, "Output impedance design of parallel-connected ups inverters with wireless load-sharing control", IEEE Transactions on Industrial Electronics, vol.52, no.4, pp. 1126-1135, Aug/2005.

[6] K. Brabandere, B. Bolsens, J. V. den Keybus, A. Woyte, J. Driesen, R. Belmans, "A voltage and frequency droop control method for parallel inverters", IEEE Transactions on Power Electronincs, vol. 22, no. 4, pp. 1107-1115, Jul/2007.

[7] R. Majumder, B. Chaudhuri, A. Ghosh, R. Majumder, G. Ledwich, F. Zare, "Improvement of stability and load sharing in an autonomous microgrid using supplementary droop control loop", IEEE Transactions on Power Systems, vol. 25, no. 2, pp. 796-808, May/2010.

[8] G. M. S. Azevedo, F. Bradaschia, M. C. Cavalcanti, 
F. A. S. Neves, J. Rocabert, P. Rodriguez, "Safe transient operation of microgrids based on master-slave configuration", in 2011 IEEE Energy Conversion Congress and Exposition, pp. 2191-2195, 2011.

[9] J. Hu, J. Zhu, D. G. Dorrell, J. M. Guerrero, "Virtual flux droop method - a new control strategy of inverters in microgrids", IEEE Transactions on Power Electronincs, vol. 29, no. 9, pp.4704-4711, Sept/2014.

[10] J. M. Guerrero, J. C. Vasquez, J. Matas, L. G. Vicuña, M. Castilla, "Hierarchical control of droop-controlled ac and dc microgrids - a general approach toward standardization", IEEE Transactions on Industrial Electronics, vol. 58, no. 1, pp. 158-172, Jan/2011.

[11] J. M. Guerrero, M. Chandorkar, T. Lee, P. C. Loh, "Advanced control architectures for intelligent microgrids - part I: Decentralized and hierarchical control", IEEE Transactions on Industrial Electronics, vol.60, no.4, pp. 1254-1262, Apr/2013.

[12] J. A. P. Lopes, C. L. Moreira, A. G. Madureira, "Defining control strategies for microgrids islanded operation", IEEE Transactions on Power Systems, vol. 21, no.2, pp. 916-924, May/2006.

[13] C. L. Moreira, F. O. Resende, J. A. P. Lopes, "Using low voltage microgrids for service restoration", IEEE Transactions on Power Systems, vol. 22, no.1, pp. 395403, Feb/2007.

[14] J. Rocabert, G. M. S. Azevedo, A. Luna, J. M. Guerrero, I. Candela, P. Rodríguez, "Intelligent connection agent for three-phase grid-connected microgrids", IEEE Transactions on Power Electronincs, vol.26, no.10, pp. 2993-3005, Oct/2011.

[15] G. M. S. Azevedo, M. C. Cavalcanti, F. A. S. Neves, L. R. Limongi, F. Bradaschia, "A control of microgrid power converter with smooth transient response during the change of connection mode", in 2013 Brazilian Power Electronics Conference, pp. 1008-1015, 2013.

[16] J. Rocabert, G. M. S. Azevedo, I. Candela, R. Teoderescu, P. Rodriguez, I. Etxebarria-Otadui, "Microgrid connection management based on an intelligent connection agent", in 2010 Conference on IEEE Industrial Electronics Society, pp. 3028-3033, 2010.

[17] A. Timbus, M. Liserre, R. Teodorescu, P. Rodriguez, F. Blaabjerg, "Evaluation of current controllers for distributed power generation systems", IEEE Transactions on Power Electronincs, vol. 24, no. 3, pp. 654-664, Mar/2009.

[18] D. N. Zmood, D. G. Holmes, "Stationary frame current regulation of pwm inverters with zero steady-state error", IEEE Transactions on Power Electronincs, vol. 18, no. 3, pp. 814-822, May/2003.

[19] Y. Sato, T. Ishizuka, K. Nezu, T. Kataoka, "A new control strategy for voltage-type pwm rectifiers to realize zero steady-state control error in input current", IEEE Transactions on Industry Applications, vol. 34, no. 3, pp. 480-486, May/Jun/1998.

[20] G. M. S. Azevedo, F. A. S. Neves, M. C. Cavalcanti, L. R. Limongi, F. Bradaschia, "Fault detection system for distributed generation converters", in 2011 Brazilian

Power Electronics Conference, pp. 320-327, 2011.

[21] G. M. S. Azevedo, M. C. Cavalcanti, F. A. S. Neves, F. Bradaschia, "A fast fault detection scheme for power converters in distributed generation systems", Eletronica de Potencia SOBRAEP, vol. 17, no.2, pp. 546-554, Mar/May/2012.

[22] P. Rodriguez, A. V. Timbus, R. Teodorescu, M. Liserre, F. Blaabjerg, "Flexible active power control of distributed power generation systems during grid faults", IEEE Transactions on Industrial Electronics, vol. 54, no. 5, pp. 2583-2592, Oct/2007.

[23] P. Rodriguez, A. Luna, M. Ciobotaru, R. Teodorescu, F. Blaabjerg, "Advanced grid synchronization system for power converters under unbalanced and distorted operating conditions", in 2006 Conference on IEEE Industrial Electronics Society, pp. 5173-5178, 2006.

[24] P. Rodriguez, A. Luna, R. S. Muñoz-Aguilar, I. Etxeberria-Otadui, R. Teodorescu, F. Blaabjerg, "A stationary reference frame grid synchronization system for three-phase grid-connected power converters under adverse grid conditions", IEEE Transactions on Power Electronincs, vol. 27, no. 1, pp. 99-112, Jan/2012.

\section{BIOGRAPHIES}

Gustavo Medeiros de Souza Azevedo was born in Belo Jardim, Brazil, in 1981. He received the B.S., M.S. and Ph.D. degrees in electrical engineering from the Federal University of Pernambuco in 2005, 2007 and 2011, respectively. He worked as a visiting scholar at the Polytechnical University of Catalunya, Barcelona, Spain, from 2008 to 2009. Since January 2014, he is working as an associate professor in the Department of Electrical Engineering at the Federal University of Pernambuco. His research interests are renewable energy systems and microgrids.

Marcelo Cabral Cavalcanti was born in Recife, Brazil, in 1972. He received the B.S. degree in electrical engineering in 1997 from the Federal University of Pernambuco, Recife, Brazil, and the M.S. and Ph.D. degrees in electrical engineering from the Federal University of Campina Grande, Campina Grande, Brazil, in 1999 and 2003, respectively. He worked as a visiting scholar at Center for Power Electronics Systems, Virginia Polytechnic Institute and State University, Blacksburg - USA, from Oct/2001 to Aug/2002, and at Alcala University - Spain, from Set./2012 to Aug./2013. Since 2003, he has been at the Department of Electrical Engineering, Federal University of Pernambuco, where he is currently a Professor of Electrical Engineering. His research interests are renewable systems and power quality.

Francisco de Assis dos Santos Neves was born in Campina Grande, Brazil, in 1963. He received the B.S. and M.Sc. degrees in electrical engineering from the Federal University of Pernambuco, Recife, Brazil, in 1984 and 1992, respectively, and the Ph.D. degree in electrical engineering from the Federal University of Minas Gerais, Belo Horizonte, Brazil, in 1999. $\mathrm{He}$ worked as a visiting scholar at Georgia Institute of Technology - USA, during 1999, and at Alcala University - Spain, from Feb./2008 to Jan./2009. Since 1993, he has 
been with the Department of Electrical Engineering, Federal University of Pernambuco, where he is currently a Professor of Electrical Engineering. His research interests are power electronics, renewable energy systems, power quality and grid synchronization methods.

Leonardo Rodrigues Limongi was born in Recife, Brazil, in 1978. He received the M.Sc. degree in electrical engineering from the Universidade Federal de Pernambuco, Recife, Brazil, in 2006, and the Ph.D. degree from the Politecnico di Torino, Turin, Italy, in 2009. Since 2010, he has been at the Electrical Engineering Department, Universidade Federal de Pernambuco, where he is currently a Professor of Electrical Engineering. He is the author of more than 20 papers published in international conference proceedings and technical journals. His research interests include the fields of power electronics dedicated to power conditioning systems and distributed generation.

Fabrício Bradaschia was born in São Paulo, Brazil, in 1983. He received the B.Sc., M.Sc. and Ph.D. degrees in electrical engineering from the Federal University of Pernambuco, Recife, Brazil, in 2006, 2008 and 2012, respectively. From August 2008 to August 2009, he worked as a visiting scholar at the University of Alcalá, Madrid, Spain. Since October 2013, he is working as an associate professor in the Department of Electrical Engineering at the Federal University of Pernambuco. His research interests are application of power electronics in renewable energy systems and power quality, including the study of pulse-width modulation, converter topologies and grid synchronization methods. 